\title{
Rotation effect on near-wall turbulence statistics and flow structures
}

\author{
LIU Nansheng, LU Xiyun \& ZHUANG Lixian \\ Department of Mechanics and Mechanical Engineering, University of Science and Technology of China, \\ Hefei 230026, China \\ Correspondence should be addressed to Lu Xiyun (email: xlu@ustc.edu.cn) \\ Received November 9, 2004
}

\begin{abstract}
Large eddy simulation (LES) of turbulent flow through an axially rotating pipe, coupled with nonlinear dynamic subgrid-scale (SGS) model, is carried out to investigate rotation effect on the near-wall turbulence characteristics and flow structures. In the rotating turbulent pipe flow, it is found that the tendency towards a relaminarized flow appears and the axial velocity fluctuation is suppressed; however, the azimuthal fluctuation is enhanced due to the presence of the pipe wall rotation. The joint probability density function (joint PDF) of the velocity fluctuations and the probability density function (PDF) of the helicity fluctuation are analyzed in detail. It is revealed that the resolved Reynolds stress and helicity fluctuation in the wall region are closely related to the correlation between the velocity and vorticity fluctuations and affected significantly by the rotation-induced azimuthal mean flow. Further, the budgets of resolved Reynolds stresses indicate that the rotation effect is responsible for the more active turbulent energy redistribution and the production of the azimuthal turbulence fluctuation. The near-wall inclined streaky structures with respect to the axial direction are ascribed to the spiral motion of the fluid induced by the rotating pipe. The turbulence characteristics revealed in this study are of great help for the understanding of physical fundamentals in the rotating turbulent flows and for the development of reliable turbulence model.
\end{abstract}

Keywords: large eddy simulation (LES), dynamic subgrid-scale (SGS) model, rotating turbulent flow, coherent structures.

DOI: 10.1360/04yw0055

Turbulent flow in an axially rotating pipe, involving complicated physical mechanism of turbulence, is a typical problem for the study of rotating turbulent flow. The pipe rotation induces two effects on the flow. One is the stabilizing effect due to the centrifugal and Coriolis forces, which accounts for the relaminarization of the turbulence ${ }^{[1-3]}$ and the reduction of the friction coefficient at the pipe wall. The behavior is also related to the wall streaks inclining to the azimuthal direction due to the rotation effect ${ }^{[4]}$. The momentum and energy transfer in the rotating pipe flow is remarkably suppressed ${ }^{[5]}$. The other is the destabilizing effect due to the tangential shear stress caused by the rotating 
pipe wall ${ }^{[5,6]}$. The tangential wall shear stress subjected to the rapid rotation speed contributes to the generation of turbulent kinetic energy.

Some work on the rotating pipe flow has been carried out to mainly deal with the turbulence statistics rather than with the near-wall coherent structures, which are linked to the wall shear stress. As is well known, the near-wall vortical structures are closely relevant to the burst events in the boundary layer and to the generation of the friction force (or Reynolds stress) in the wall region ${ }^{[7]}$. The joint PDF and PDF of turbulence fluctuations are well accepted to be helpful to reveal the motion of small structures in turbulence ${ }^{[8,9]}$. Meanwhile, the budgets of Reynolds stress not only reflect the rotation effect on the near-wall vortical structures ${ }^{[10]}$, but also provide detailed information of the dynamic process of the wall turbulence, e.g. production, dissipation, and redistribution of turbulent kinetic energy ${ }^{[11]}$. Therefore, it is needed to identify the rotational dependence of the drag reduction on the turbulence coherent structures in the wall region of the rotating pipe based on the joint PDF and PDF of turbulence fluctuations. It is also interesting to explore the mechanism of turbulent transport due to the fluctuating velocity by use of the budgets of Reynolds stress. In the wall-bounded turbulent flow, the region with high-level helicity fluctuation is usually characterized by weak nonlinear interaction between the motions of large and small scales ${ }^{[12]}$, which results in the more ordered near-wall vortical structures and accounts for the drag reduction in the rotating pipe flow $^{[13]}$. As found by Orlandi ${ }^{[14]}$, the PDF distributions of the helicity and Lamb vector fluctuations are of great importance in the prediction of the near-wall drag force.

Little work on the turbulent flow in an axially rotating pipe has been performed by means of LES technique. Yang ${ }^{[15]}$ carried out the LES coupled with the eddy viscosity model $^{[16]}$ and dynamic SGS model ${ }^{[17]}$ to show that obvious deviations between calculated results and experimental data occur. This fact is due to the inherent shortcomings of the eddy viscosity ${ }^{[18]}$ and the inconsistency of the dynamic SGS model with the constraint to the SGS stress closure for the rotating turbulence, i.e. the principle of asymptotic material indifference $(\mathrm{AMFI})^{[19,20]}$.

In this study, the LES combined with nonlinear dynamic SGS model, which satisfies the principle of AMFI, is performed to investigate rotating turbulent pipe flow at high Reynolds number and rotation speed. In the nonlinear dynamic SGS model, the resolved rotation tensor $\bar{\omega}_{i j}$ and the strain tensor $\bar{s}_{i j}$ are introduced to compose the second-order nonlinear terms, which represent implicitly the coupled effect of the rotation and shear on the rotating turbulent flow. Further, some typical quantities, including the joint PDF of the velocity fluctuations, the PDF of the helicity fluctuation, and the budgets of resolved Reynolds stress, are analyzed in detail to investigate the near-wall turbulence statistics and flow structures in the rotating pipe flow.

\section{Mathematical formulation}

The governing equations for the LES of the turbulent rotating pipe flow are the 
three-dimensional filtered Navier-Stokes equations. To non-dimensionalize the governing equations, as shown in fig. 1, the bulk mean velocity $U_{m}$ and the pipe radius $R$ are used as velocity and length scales, respectively. Then, the non-dimensional equations are given as

$$
\begin{gathered}
\frac{\partial \bar{v}_{i}}{\partial x_{i}}=0 \\
\frac{\partial \bar{v}_{i}}{\partial t}+\frac{\partial\left(\bar{v}_{i} \bar{v}_{j}\right)}{\partial x_{j}}=-\frac{\partial \bar{p}}{\partial x_{i}}+\frac{1}{\operatorname{Re}} \frac{\partial^{2} \bar{v}_{i}}{\partial x_{j} \partial x_{j}}-\frac{\partial \tau_{i j}}{\partial x_{j}}
\end{gathered}
$$

where the overbar denotes the resolved variable, $\tau_{i j}=\overline{v_{i} v_{j}}-\bar{v}_{i} \bar{v}_{j}$ represents the SGS stress and needs to be modeled by SGS model. The Reynolds and rotation numbers are defined as $R e=2 U_{m} R / v$ and $N=\Omega R / U_{m}$, respectively, with $\Omega$ being the angular speed of the pipe.

Here, eqs. (1) and (2) are numerically solved in the cylindrical coordinate. Since the fully developed turbulent pipe flow is considered here, periodic conditions are thus employed in the axial and azimuthal directions. No-slip and no-penetration conditions are imposed at the pipe wall, i.e. the rotational speed of the pipe wall is fixed as $\bar{v}_{\theta}=N$, while the other two components of velocity are zero.

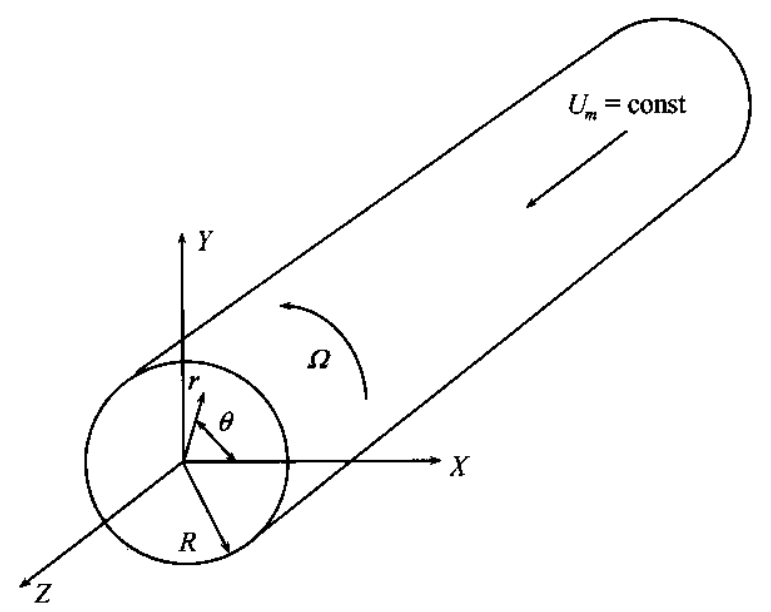

Fig. 1. Sketch of the rotating turbulent pipe flow.

\section{Nonlinear SGS model for rotating turbulent flow}

Nonlinear dynamic SGS model, which is consistent with the principle of $\mathrm{AMFI}^{[21]}$, is used in this calculation and given as in the inertial frame 


$$
\begin{aligned}
\tau_{i j}-\frac{1}{3} \tau_{k k} \delta_{i j}= & -\lambda v_{a} \bar{s}_{i j}+v_{b}\left(\bar{s}_{i k} \bar{\omega}_{k j}+\bar{s}_{j k} \bar{\omega}_{k i}\right)+v_{c}\left(\bar{s}_{i k} \bar{s}_{k j}-\frac{1}{3} \bar{s}_{k l} \bar{s}_{k l} \delta_{i j}\right) \\
& -v_{b}\left(\bar{\omega}_{i k} \bar{\omega}_{k j}-\frac{1}{3} \bar{\omega}_{l k} \bar{\omega}_{k l} \delta_{i j}\right)
\end{aligned}
$$

where the resolved strain tensor $\bar{s}_{i j}$ and rotation tensor $\bar{\omega}_{i j}$ read as

$$
\bar{s}_{i j}=\frac{1}{2}\left(\frac{\partial \bar{v}_{i}}{\partial x_{j}}+\frac{\partial \bar{v}_{j}}{\partial x_{i}}\right), \quad \bar{\omega}_{i j}=\frac{1}{2}\left(\frac{\partial \bar{v}_{j}}{\partial x_{i}}-\frac{\partial \bar{v}_{i}}{\partial x_{j}}\right)
$$

It is worthwhile to note that the rotation number $N$ represents implicitly the rotation effect of the rotating pipe on the SGS stress. In particular, the rotation tensor $\bar{\omega}_{i j}$, which contains the axial vorticity component induced by the rotating pipe wall, has a significant influence on the SGS stress. It has been verified that the nonlinear SGS model in inertial frame can be obtained from its corresponding form in rotating frame by taking an orthogonal rotational frame transformation ${ }^{[21]}$. The model coefficients $v_{a}, v_{b}$ and $v_{c}$ can be determined dynamically based on the procedure proposed by Germano et al. ${ }^{[17]}$.

\section{Numerical method}

To perform LES calculation, the fractional-step method, developed by Verzicco and Orlandi ${ }^{[22]}$, was employed to solve the governing equations. The spatial derivatives are discretized by the second-order central different scheme. The convection and viscous terms are treated by Adams-Bashforth and Crank-Nicholson schemes, and the third-order Runge-Kutta scheme is used for the time advancement. Based on our previous work ${ }^{[23-26]}$, it is confirmed that the numerical method used in the present LES is reliable. Some typical validation is also shown in the following.

\section{Results and discussion}

Here, the Reynolds number is chosen as $R e=20000$ and the rotation number varies from 0 to 4 . According to previous finding ${ }^{[4]}$, the rotation effect gives rise to the large-scale structures in the central region of the pipe, aligned to the axial direction; the scale of these structures becomes larger as the rotation number increases. The pipe length is chosen as 25 times of the pipe radius, which is confirmed to be a sufficient length to contain the largest coherent structure aligned with the axial direction based on calculation test. The grid system of $65 \times 73 \times 97$ is adopted to resolve the computational domain in the azimuthal, radial and axial directions, respectively. To achieve a fine grid resolution near the wall, a stretching transformation in the radial direction is employed. Uniform grid is employed in the azimuthal and axial directions.

\subsection{Validation}

In fig. 2, some typical numerical results are demonstrated to examine the perform- 
ance of the computational code. For simplicity, in the following discussion, the results obtained by use of the eddy viscosity model ${ }^{[16]}$, the dynamic SGS model and the nonlinear SGS model are denoted by SSM, GSM and NSM respectively, while experimental data $^{[5]}$ by EXP. The resolved mean velocity $\left\langle\bar{v}_{i}\right\rangle$ is the ensemble average of the filtered velocity, and the velocity fluctuation is defined as $\bar{v}_{i}=\bar{v}_{i}-\left\langle\bar{v}_{i}\right\rangle$.
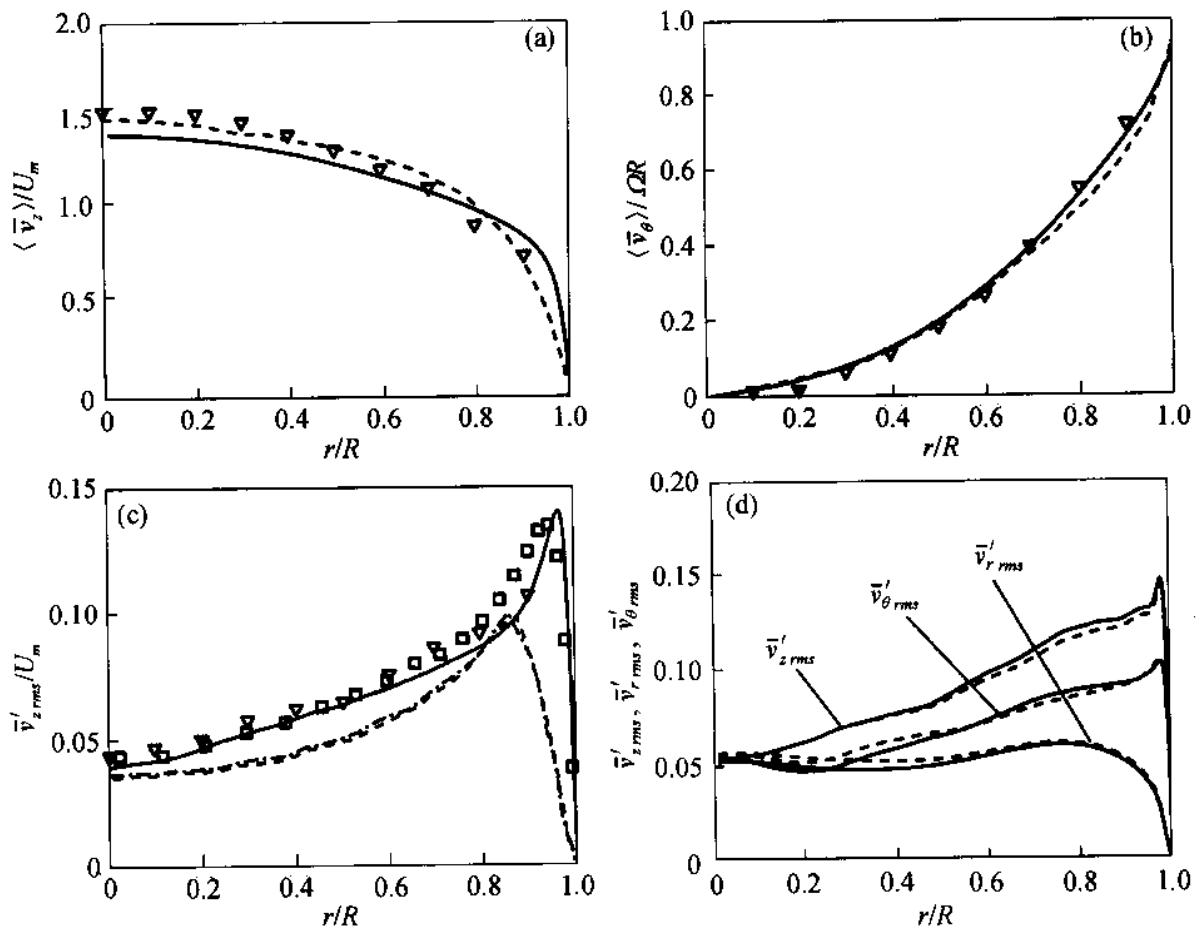

Fig. 2. Results of the rotating turbulent pipe flow at $N=1.0$. (a) Axial mean velocity; (b) azimuthal mean flow; (c) axial turbulence intensity; here, $\nabla$, experimental data (EXP); $\square$, channel flow data; dashed line: eddy viscosity model (SSM), dash-dot line: dynamics SGS model (GSM), solid line: nonlinear SGS model (NSM); (d) turbulence intensity calculated by different grid sizes, solid line: $65 \times 73 \times 97$, dashed line: $97 \times 97 \times 128$.

The profiles of the axial mean velocity are shown in fig. 2(a). Although the mean velocity distributions predicted numerically are slightly smaller than the experimental data, calculated results compare quite well with each other. The profiles of the azimuthal mean velocity in fig. 2(b) agree well with each other. As shown in fig. 2(c), the axial turbulence intensity $\bar{v}_{z r m s}$ predict ed by NSM compares fairly well with the experimental data; however, it is evident that deviation between the results calculated by SSM and GSM and the experimental data occurs apparently. A peak value of $\bar{v}_{z r m s}$ by NSM appears at $\delta / R=0.04$ from the wall, which is quite well consistent with the location of the streamwise turbulence intensity in the channel flow ${ }^{[27]}$, but different from $\delta / R=0.14$ and 0.12 calculated by SSM and GSM. This fact indicates that the NSM is more reliable 
to predict the behavior of the wall shear turbulence. Meanwhile, to verify that the computed results are independent of the grid sizes, the turbulence intensities calculated by different grid numbers are shown in fig. 2(d); both the results agree well with each other.

\subsection{Mean velocity and turbulence intensities}

In fig. 3(a), the axial mean velocity $\left\langle\bar{v}_{z}\right\rangle$, scaled by the friction velocity, increases in the central region of the pipe with the increase of the rotation number $N$, indicating a tendency of turbulence towards the laminar flow ${ }^{[4]}$. In the non-rotating case (i.e. $N=0$ ), there exists apparently the linear and logarithmic region with a narrow buffer layer. The axial mean velocity in the logarithm region follows the relation, $\left\langle\bar{v}_{z}\right\rangle^{+}=(1 / K) \log y^{+}+B$, where the constants $K$ and $B$ are equal to 0.41 and 5.5, respectively. As $N$ increases, $\left\langle\bar{v}_{z}\right\rangle^{+}$still satisfies the linear law distribution in the sublayer, while deviates from the original logarithm law in the center of the pipe. Based on the distributions of $\left\langle\bar{v}_{z}\right\rangle^{+}$, it can be seen that the constant $K$, at $N=0.5,1,2$ and 4 , becomes $0.35,0.25,0.16$ and 0.1 , while the constant $B$ is $4.5,1.5,1.2$ and -6 , respectively. Moreover, fig. 3(a) reflects the diminishing of the logarithmic region and the thickening of the buffer layer. This feature was also observed in the rotating pipe flow at low Reynolds number ${ }^{[4]}$.
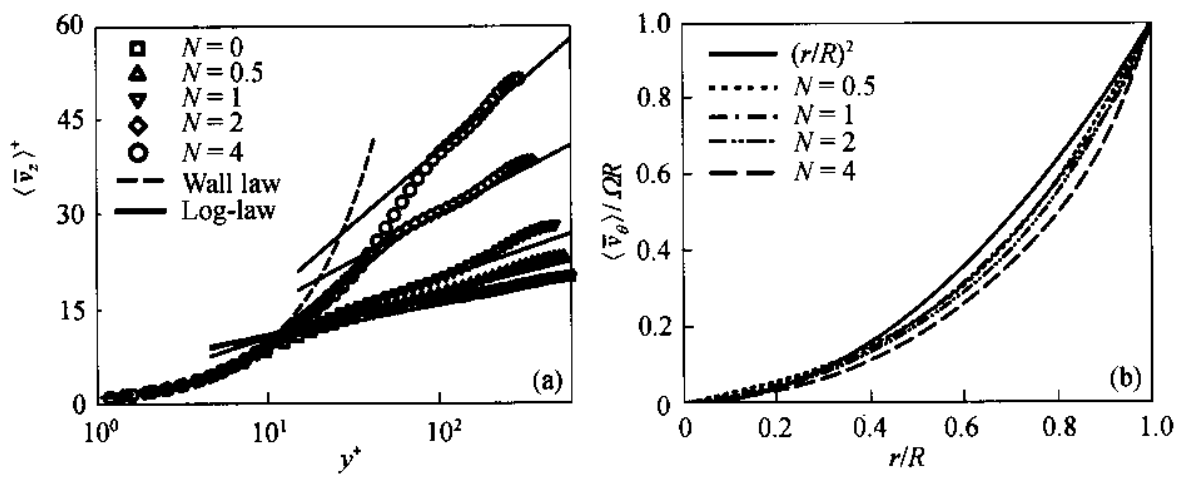

Fig. 3. Mean velocities at different rotation numbers. (a) Axial; (b) azimuthal component.

Fig. 3(b) shows the azimuthal mean velocity $\left\langle\bar{v}_{\theta}\right\rangle$ in the rotating pipe. Reich and Beer $^{[6]}$ indicated that $\left\langle\bar{v}_{\theta}\right\rangle$ tends towards the parabolic distribution when $N$ varies from 0.5 to 2.0 and the profile of $\left\langle\bar{v}_{\theta}\right\rangle$ is approximately independent of $N$, if scaled by the rotational speed of the pipe wall $V_{\theta w}$, i.e. $V_{\theta w}=\Omega R$. Similarly, Kikuyama ${ }^{[28]}$ confirmed experimentally that $\left\langle\bar{v}_{\theta}\right\rangle$ is characterized approximately by a parabolic distribution, i.e. $\left\langle\overline{v_{\theta}}\right\rangle=(r / R)^{2} V_{\theta w}$. Speziale et al. ${ }^{[29]}$ attributed this feature to the existence of secondary flow induced by the rotation effect. As shown in fig. 3(b), the enhancement of the wall shear effect is linked to the increase of the azimuthal mean velocity. 
The profiles of turbulence intensities are depicted in fig. 4. As $N$ increases, $\bar{v}_{z r m s}$ decreases obviously in the sublayer, while slightly increases in the outer region away from the pipe wall. Orlandi and Fatical ${ }^{[4]}$ observed that the peak value of $\bar{v}_{z r m s}$ near the wall is smoothed at $N=2$ with low Reynolds number $\mathrm{Re}=4900$. However, this feature was not observed experimentally in the rotating pipe flow at high Reynolds number ${ }^{[28]}$. So, the smoothing of the peak value of $\bar{v}_{z r m s}$ is asserted to only exist in the case of low Reynolds number flow ${ }^{[4]}$. At $N=0.5$ and 1 , as shown in fig. 4(b), the radial turbulence intensity $\bar{v}_{r r m s}$ exhibits slight reduction in the region of $r / R>0.3$ but small increase in the center of the pipe, compared to the case at $N=0$. As $N$ increases to $4, \bar{v}_{r r m s}$ is augmented slightly nearly over the whole pipe. The trend of $\bar{v}_{r r m s}$ in fig. 4(b) indicates that the radial turbulence intensity is suppressed as $N \leqslant 2$, but enhanced when $N$ increases further. As shown in fig. 4(c), the azimuthal turbulence intensity $\bar{v}_{\theta r m s}$ exhibits a monotonic increase with the increase of $N$. In the pure shear pipe flow, the generation of the azimuthal turbulence fluctuation is mainly due to the splattering effect linked to the high-speed fluid rushing into the sublayer ${ }^{[7]}$. However, the rotation effect in the rotating pipe gives rise to other mechanism to generate the azimuthal fluctuation, i.e. the shear effect due to the azimuthal mean flow. The azimuthal shear effect becomes more

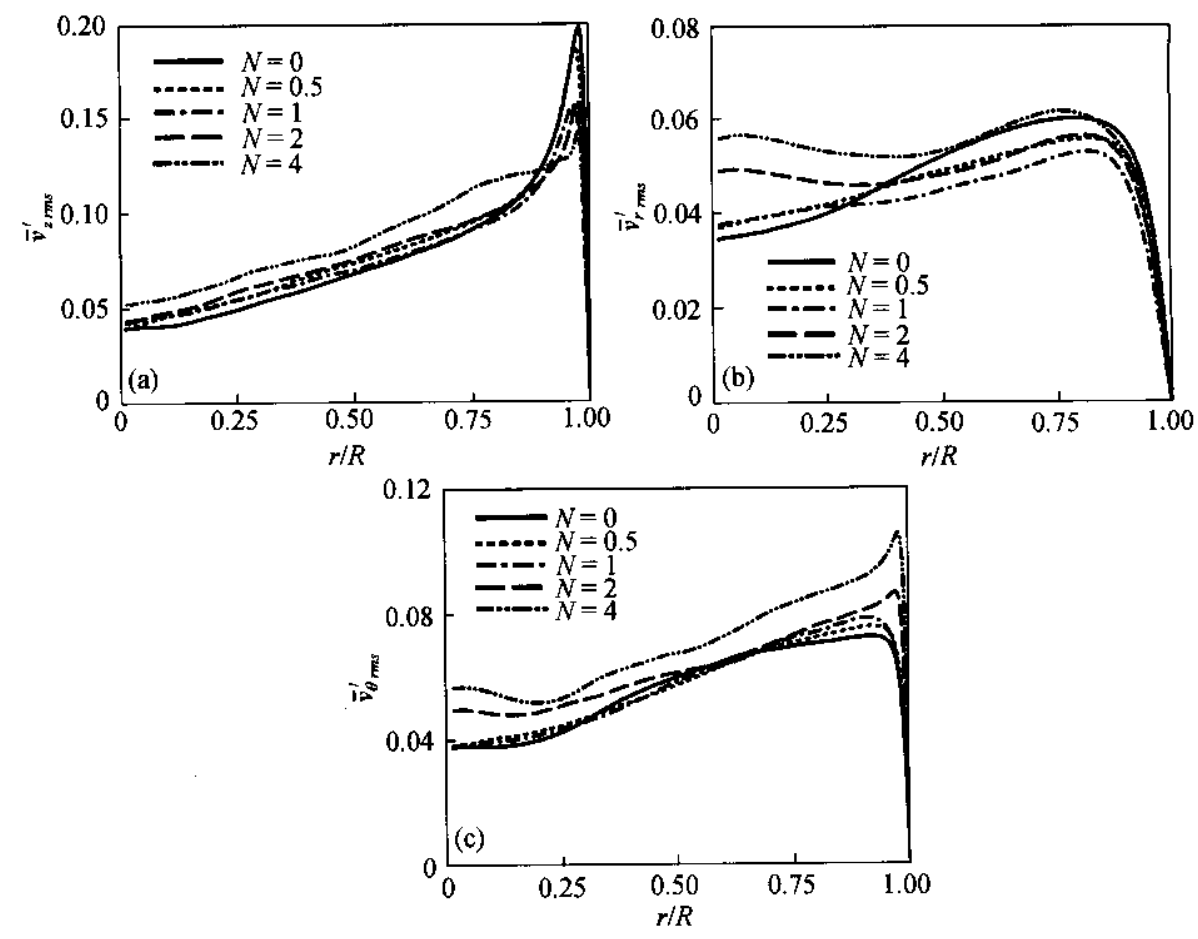

Fig. 4. Turbulence intensities: (a) axial; (b) radial; (c) azimuthal component. 
inten sive at higher $N$, and accounts for the major generation of the azimuthal velocity fluctuation.

\subsection{Reynolds stresses and joint PDF of velocity fluctuations}

Fig. 5 shows the distributions of the resolved Reynolds stresses. It is observed in fig. 5(a) that $\left\langle\bar{v}_{r} \bar{v}_{z}\right\rangle$ is suppressed when $N$ varies from 0 to 1 and enhanced at $N=2$ and 4 . Compared to the case $N=0$, the drag reduction, corresponding to the decrease of $\left\langle\bar{v}_{r} \bar{v}_{z}\right\rangle$, is identified in the rotating pipe flow. This trend of $\left\langle\bar{v}_{r} \bar{v}_{z}\right\rangle$ results from the stabilizing effect induced by centrifugal force that suppresses the ejection and sweep events linked to the turbulence coherent structures, which are responsible for the generation of $\left\langle\bar{v}_{r} \bar{v}_{z}\right\rangle$ in the wall region ${ }^{[7]}$. The non-zero distribution of $\left\langle\bar{v}_{\theta} \bar{v}_{r}\right\rangle$ arises in the rotating pipe, as shown in fig. 5(b), which is at least one order lower than $\left\langle\bar{v}_{r} \bar{v}_{z}\right\rangle$ and exhibits remarkable peak value in the wall region at $N=2$ and 4 . Similar to the mechanism of the contribution of $\partial\left\langle\bar{v}_{z}\right\rangle / \partial r$ to the generation of $\left\langle\bar{v}_{r} \bar{v}_{z}\right\rangle$, the increase of $\partial\left\langle\bar{v}_{\theta}\right\rangle / \partial r$ indeed contributes to the near-wall generation of $\left\langle\bar{v}_{\theta} \bar{v}_{r}\right\rangle$, hence causes a notable increase of $\bar{v}_{\theta r m s}$ in the wall region, as shown in fig. 4(c), since $\left\langle\bar{v}_{\theta} \bar{v}_{r}\right\rangle$ involves the production of the azimuthal turbulent kinetic energy $\left\langle\overline{v_{\theta}} \bar{v}_{\theta}\right\rangle{ }^{[10]}$. The peak value of $\left\langle\bar{v}_{\theta} \bar{v}_{z}\right\rangle$ gradually increases with the increase of $N$ in fig. 5(c), and becomes comparable to $\left\langle\bar{v}_{r} \bar{v}_{z}\right\rangle$ in the wall region at $N=2$ and 4 . According to Orlandi and Fatica ${ }^{[4]}$, the trend of $\left\langle\begin{array}{ll}\bar{v}_{\theta} & \bar{v}_{z}\end{array}\right\rangle$ is ascribed to the tilting of the near-wall vortical structures, which leads to the more intensive correlation between $\bar{v}_{\theta}$ and $\bar{v}_{z}$. The overview of fig. 5(b) and (c) is the enhanced correlations of $\bar{v}_{\theta}$ with both $\bar{v}_{r}$ and $\bar{v}_{z}$, especially in strong rotation cases. These enhanced correlations reasonably imply the recovery of the Reynolds stress $\left\langle\bar{v}_{r} \bar{v}_{z}\right\rangle$ in the cases, e.g. $N=2$ and 4 , as observed in fig. 5(a).

Further, to understand the rotation effect on the correlations of the near-wall turbulence fluctuations, the joint PDFs of velocity fluctuations at $y^{+}=5.2$ for $N=0$ and 4 are shown in figs. 6-8, where the velocity fluctuations are scaled by their local root-mean-square (rms) values in the $(\theta, z)$ plane. As shown in fig. 6(a), it is suggested that the sweep and ejection events, contributing to the generation of $\left\langle\bar{v}_{r} \bar{v}_{z}\right\rangle$, lie in the fourth and second quadrants of the joint PDF, respectively. At $N=0$, the predominance of the joint PDF in the second quadrant over that in the fourth quadrant indicates that the turbulence sweep events, compared to the ejection events, occur actively in the rotating pipe flow. Thus, the generation of $\left\langle\bar{v}_{r} \bar{v}_{z}\right\rangle$ is mainly due to the near-wall fluid lifting away from the sublayer. As shown in fig. 6(b) for $N=4$, it is evident that the joint PDF is relatively enhanced in the second quadrant and suppressed in the fourth quadrant. This feature means that the probability of the ejection event increases in the wall region and 
the probability of the sweep event decreases relatively.
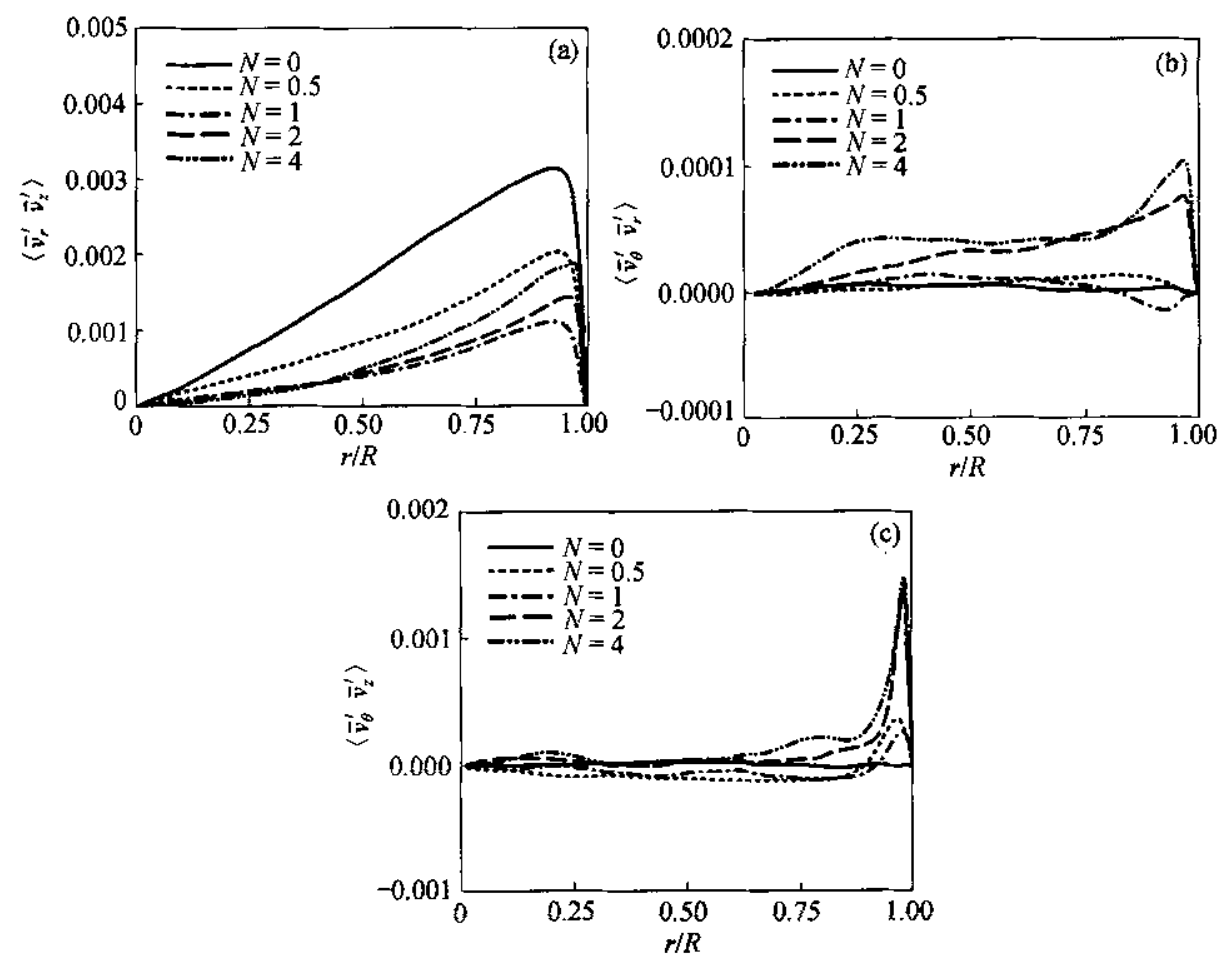

Fig. 5. Resolved Reynolds stresses: (a) $\left\langle\bar{v}_{r} \bar{v}_{z}\right\rangle$; (b) $\left\langle\bar{v}_{\theta} \bar{v}_{r}\right\rangle$; (c) $\left\langle\bar{v}_{\theta} \bar{v}_{z}\right\rangle$.
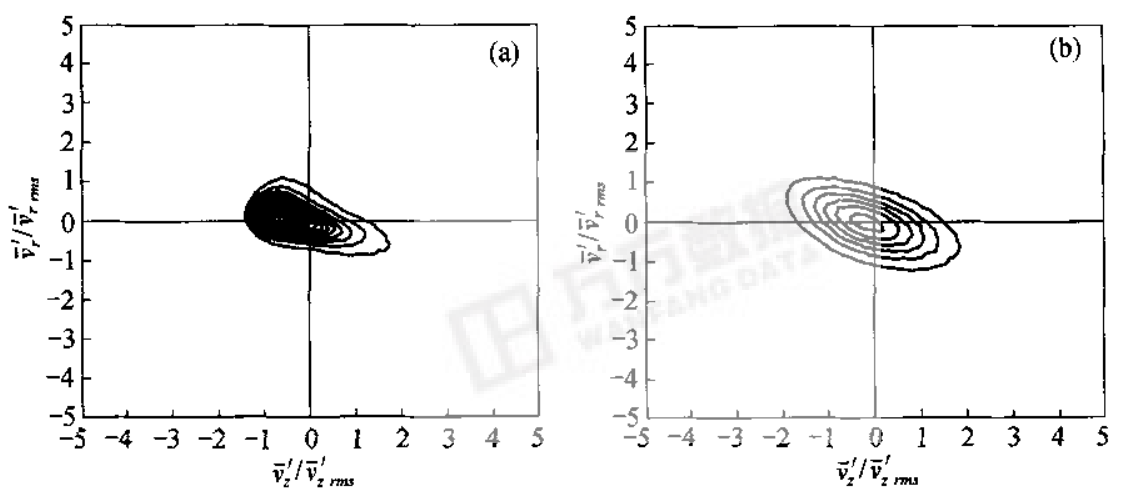

Fig. 6. Joint PDF of velocity fluctuation $\bar{v}_{z}$ and $\bar{v}_{r}$ in the $(\theta, z)$ plane at $y^{+}=5.2$ with the increment of contour $\Delta=0.0005$; (a) $N=0$; (b) $N=4$.

As shown in fig. 7(a), the joint PDF between $\bar{v}_{\theta}$ and $\bar{v}_{r}$ is symmetric about the axis $\bar{v}_{r}=0$ at $N=0$. As a result of this symmetry, net contribution due to the correlation between $\bar{v}_{\theta}$ and $\bar{v}_{r}$ to the Reynolds stress $\left\langle\bar{v}_{\theta} \bar{v}_{r}\right\rangle$ is negligible. This fact reasonably corresponds to the near zero distribution of $\left\langle\bar{v}_{\theta} \bar{v}_{r}\right\rangle$ in the non-rotating case. In the ro- 
tating pipe flow, this symmetry of the joint PDF between $\bar{v}_{\theta}$ and $\bar{v}_{r}$ no longer exists, and the joint PDFs in the first and third quadrants prevail over those in the other two quadrants, resulting in positive $\left\langle\bar{v}_{\theta} \bar{v}_{r}\right\rangle$ in the wall region, as shown in fig. 5(b) for $N=$ 4.
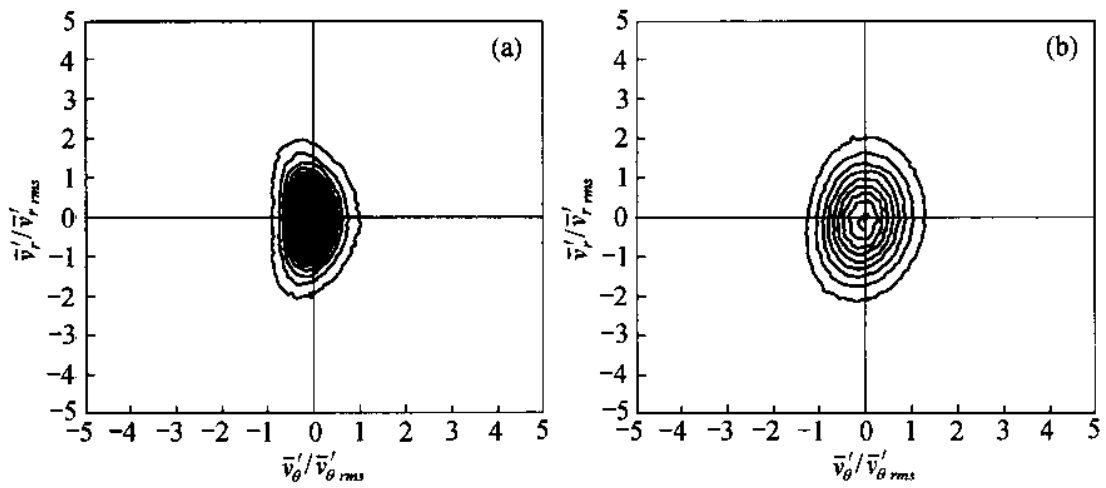

Fig. 7. Joint PDF of velocity fluctuation $\bar{v}_{\theta}$ and $\bar{v}_{r}$ in the $(\theta, z)$ plane at $y^{+}=5.2$ with the increment of contour $\Delta=0.0003$; (a) $N=0$; (b) $N=4$.

Fig. 8(a) also exhibits the symmetric distributions of the joint PDF between $\bar{v}_{\theta}$ and $\bar{v}_{z}$ about the axis $\bar{v}_{\theta}=0$ at $N=0$, which indicates a null contribution of the correlation between $\bar{v}_{\theta}$ and $\bar{v}_{z}$ to the Reynolds stress $\left\langle\bar{v}_{\theta} \bar{v}_{r}\right\rangle$, and is responsible for the near zero distribution of $\left\langle\bar{v}_{\theta} \bar{v}_{r}\right\rangle$ in the non-rotating pipe. As shown in fig. 8(a), the intensive correlation between $\bar{v}_{\theta}$ and $\bar{v}_{z}$ is mainly associated with the wall structures with $\bar{v}_{z}<0$, i.e. the low-speed streaky structures in the wall region. As the rotation is imposed to the pipe wall, the joint PDF in the first and third quadrant is enhanced, implying the stronger correlation which results in positive distribution of $\left\langle\bar{v}_{\theta} \bar{v}_{r}\right\rangle$ near the wall, as depicted in fig. 5(c).

\subsection{Helicity fluctuation and its PDF}

The profiles of the mean helicity fluctuation $\left\langle h^{\prime}\right\rangle$, which is defined as $h^{\prime}=\overrightarrow{\vec{v}} \cdot \overrightarrow{\bar{\omega}}^{\prime}$, are shown in fig. 9(a). It is evident that low-level helicity fluctuation occurs in the non-rotating pipe flow. While in the rotating case, there exists local maximum and minimum of $\left\langle h^{\prime}\right\rangle$ in the near wall region. The magnitudes of the local maximum and minimum increase significantly with the increase of $N$; it means that the near-wall turbulence becomes more helical. The distribution of $\left\langle h^{\prime}\right\rangle$, which exhibits positive value in the region close to the wall and becomes negative over most of the region away from the wall in fig. 9(a), reveals that the angle between the velocity and vorticity fluctuation vectors varies with the radial location ${ }^{[12]}$. 

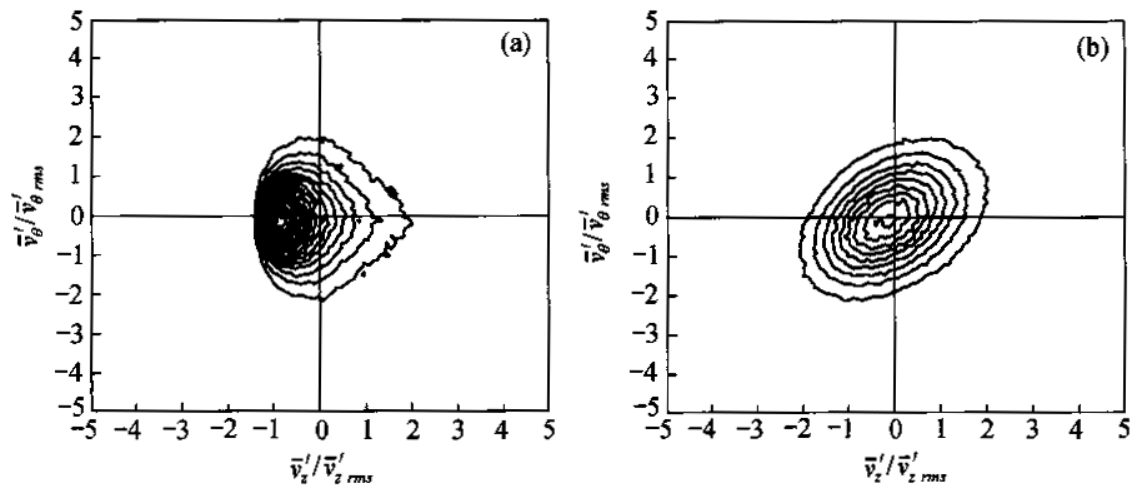

Fig. 8. Joint PDF of velocity fluctuation $\bar{v}_{z}$ and $\bar{v}_{\theta}$ in the $(\theta, z)$ plane at $y^{+}=5.2$ with the increment of contour $\Delta=0.0002$; (a) $N=0$; (b) $N=4$.
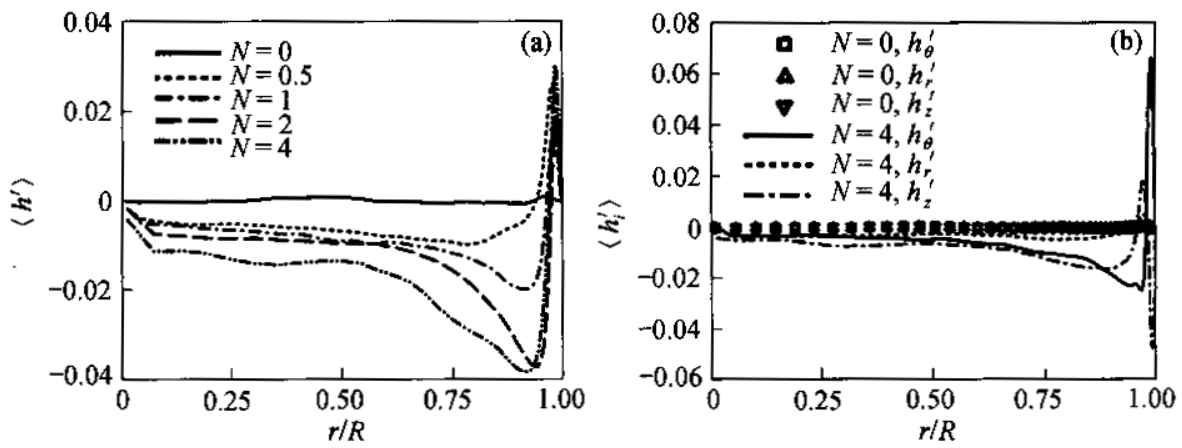

Fig. 9. Profiles of helicity fluctuation and its three parts at different rotation numbers: (a) helicity fluctuation; (b) three parts of helicity fluctuation.

Fig. 9(b) shows the profiles of three parts of helicity fluctuation $h^{\prime}$, i.e. $h_{i}^{\prime}=\bar{v}_{i} \overline{\omega_{i}}$, $i=\theta, r, z$, noting that the repeated indices do not denote the summation here. As shown in fig. 9(b), the part $\bar{v}_{\theta} \quad \bar{\omega}_{\theta}$ contributes the most to the helicity fluctuation $h^{\prime}$ among the three parts, and dominates the behavior of $\left\langle h^{\prime}\right\rangle$ in the wall region. This fact reflects that the shear effect induced by the azimuthal mean flow enhances not only the azimuthal fluctuation $\bar{v}_{\theta}$ but also the correlation between $\bar{\omega}_{\theta}$ and $\overline{v_{\theta}}$, which corresponds to the higher level of the helicity fluctuation in the near wall region.

The PDFs of $h^{\prime}$ and its three parts $h_{\theta}^{\prime}, h_{r}^{\prime}$ and $h_{z}^{\prime}$ at $y^{+}=5.2$ are shown in fig. 10, where the superscript "*" denotes the normalization by the local rms value of $|\overline{\bar{v}}| \cdot|\overline{\bar{\omega}}|$. The peak value of the PDF of $h^{\prime}$, as shown in fig. 10(a), occurs at $h^{\prime *}=0$ for $N=0$, indicating that the near-wall fluid with zero-value helicity fluctuation appears frequently. The symmetry of the PDF of $h^{\prime}$ about the axis $h^{\prime *}=0$ reasonably implies the near zero distribution of $\left\langle h^{\prime}\right\rangle$ in the wall region for $N=0$. The peak probability of 
$h^{\prime}$ decreases as $N$ increases. Meanwhile, the symmetry of the PDF of $h^{\prime}$ no longer occurs with higher probability for $h^{\prime *}>0$ prevailing over that for $h^{\prime *}<0$, causing $\left\langle h^{\prime}\right\rangle$ to be positive in the sublayer of the rotating pipe flow, as shown in fig. 9(a). Similarly, the PDF of $h_{\theta}^{\prime}$ at $N=0$ also exhibits the symmetric distribution about the axis ${h_{\theta}^{\prime *}}^{*}=0$ in fig. 10(b), which cannot be sustained due to the rotation effect. Correspondingly, the positive $h_{\theta}^{\prime}$ near the pipe wall at $N=4$ in fig. 9(b) induces a significant increase of the probability of positive $h_{\theta}^{\prime}$. As exhibited in fig. $10(\mathrm{c})$ and (d), both the PDFs of $h_{\theta}^{\prime}$ and $h_{z}^{\prime}$ are symmetric about their corresponding axes, i.e. $h_{r}^{\prime *}=0$ and $h_{z}^{\prime *}=0$, respectively, at $N=0$. Correspondingly, in the rotating case, e.g. $N=4$, the PDFs of $h_{\theta}^{\prime}$ and $h_{z}^{\prime}$ with the predominant probability for $h_{r}^{\prime}>0$ and $h_{z}^{\prime}<0$ respectively account for positive $h_{\theta}^{\prime}$ and negative $h_{z}^{\prime}$ near the wall in fig. 9(b).
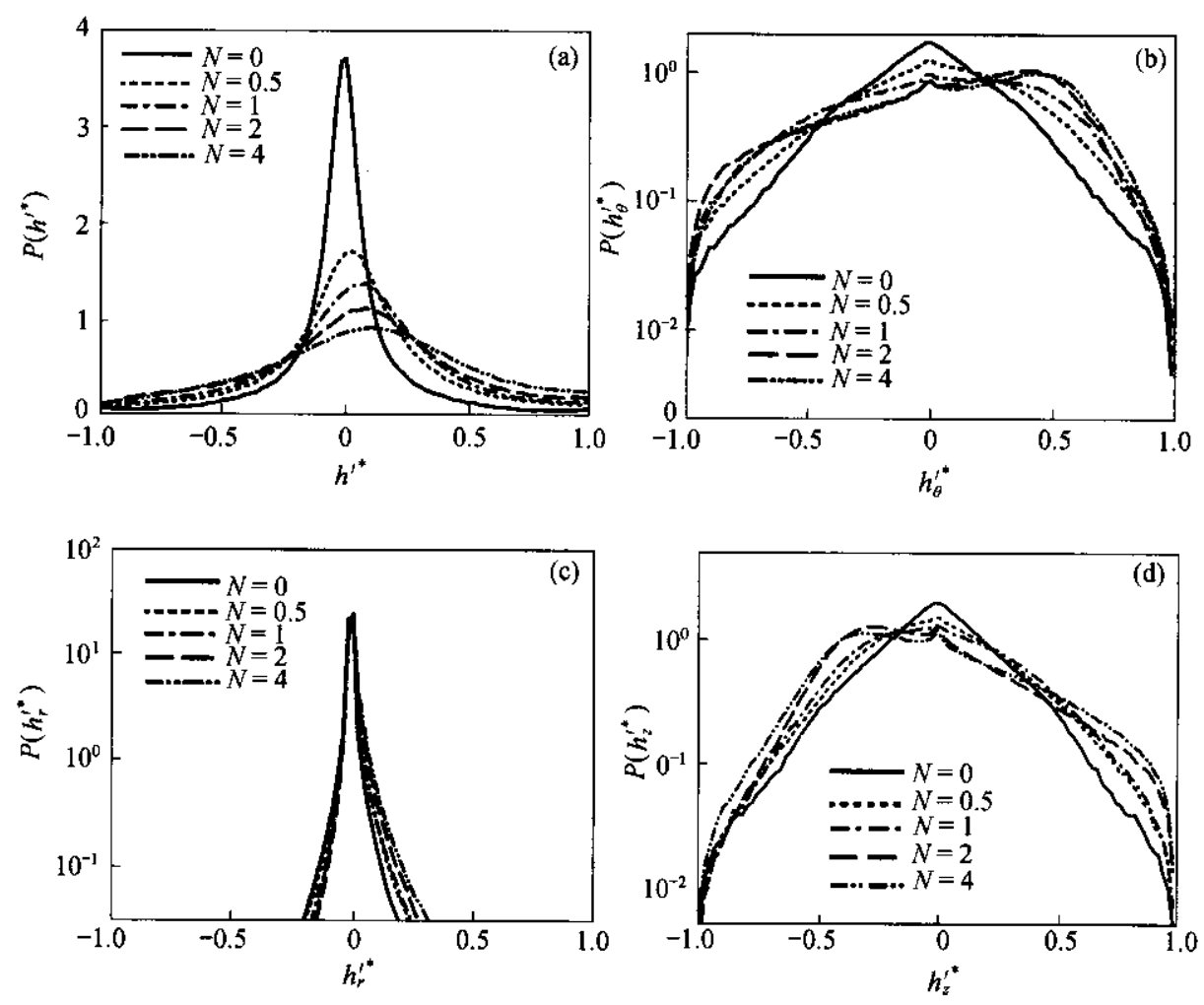

Fig. 10. PDF of helicity fluctuation and its three parts at $y^{+}=5.2:$ PDF of $h$ (a), $h_{\theta}^{\prime}(\mathrm{b}), h_{r}^{\prime}(\mathrm{c})$ and $h_{z}^{\prime}(\mathrm{d})$.

\subsection{Budgets of the resolved Reynolds stresses}

To deal with the rotation effect on the near-wall turbulence, the budgets of the resolved Reynolds stresses are examined to investigate the dynamic process of the produc- 
tion, dissipation and redistribution of turbulent kinetic energy (TKE). The transport equations of the resolved Reynolds stresses can be derived based on the ensemble average and written as

$$
\begin{aligned}
& \frac{\partial\left\langle\bar{v}_{i} \bar{v}_{j}\right\rangle}{\partial t}=-\left(\left\langle\bar{v}_{i} \bar{v}_{k}\right\rangle \frac{\partial\left\langle\bar{v}_{j}\right\rangle}{\partial x_{k}}+\left\langle\bar{v}_{j} \bar{v}_{k}\right\rangle \frac{\partial\left\langle\bar{v}_{i}\right\rangle}{\partial x_{k}}\right)-\frac{\partial\left\langle\bar{v}_{i} \bar{v}_{j} \bar{v}_{k}\right\rangle}{\partial x_{k}} \\
& -\left[\frac{\partial\left\langle P^{\prime} \bar{v}_{i}\right\rangle}{\partial x_{j}}+\frac{\partial\left\langle P^{\prime} \bar{v}_{j}\right\rangle}{\partial x_{i}}\right]+\left\langle P^{\prime}\left(\frac{\partial \bar{v}_{i}}{\partial x_{j}}+\frac{\partial \bar{v}_{i}}{\partial x_{i}}\right)\right\rangle+\left\langle\frac{\partial}{\partial x_{k}}\left[\left(R e_{\tau} v_{a}+1\right) \frac{\partial \bar{v}_{i} \bar{v}_{j}}{\partial x_{k}}\right]\right\rangle \\
& -2\left\langle\left(R e_{\tau} v_{a}+1\right) \frac{\partial \bar{v}_{i}}{\partial x_{k}} \frac{\partial \bar{v}_{j}}{\partial x_{k}}\right\rangle+H_{i j} .
\end{aligned}
$$

Here, the budget terms in eq. (5), re-scaled by $u_{\tau}^{4} / v^{[11]}$ in the following figures, are the production rate (PR, $P_{i j}$ ), turbulent diffusion (TD, $T_{i j}$ ), pressure-velocity diffusion $\left(\mathrm{PV}, \Pi_{i j}\right.$ ), pressure-strain correlation (PS, $\pi_{i j}$ ), viscous diffusion (VD, $D_{i j}$ ), and dissipation rate (DS, $\varepsilon_{i j}$ ) of the resolved Reynolds stress, respectively. The tensor $H_{i j}$ represents the sum of the additional term due to the nonlinear SGS model and the convection term due to the mean flow. It has been proved that $H_{i j}$ is negligible for the budget balance ${ }^{[7]}$.

The profiles of those terms in the $\left\langle\bar{v}_{z} \bar{v}_{z}\right\rangle$ budget at $N=0$ and 2 are exhibited in fig. 11. At $N=0$, DS and VD are dominant terms to the budget of $\left\langle\bar{v}_{z} \bar{v}_{z}\right\rangle$ in the region close to the wall. This fact suggests that the processes linked to the dissipation and viscous diffusion are considerably intensive. In the region of $8<y^{+}<30$, TD becomes more important, and PR depicts a peak value at $y^{+}=15$ approximately, corresponding to the region where the process of the turbulence production is most active. In fig. 11(b), it is seen that the rotation effect suppresses both DS and VD in the wall region, compared to the case of $N=0$. Meanwhile, PR is slightly reduced at $N=2$, together with a remarkable decrease of TD, which indicates that the process of the turbulent transport is suppressed. Interestingly, PS, which is responsible for the energy redistribution to the azimuthal and radial turbulence fluctuations, is considerably enhanced by the rotation effect.

The budget terms in the transport equation of $\left\langle\bar{v}_{\theta} \bar{v}_{\theta}\right\rangle$ are shown in fig. 12. In fig. 12(a), the budget of $\left\langle\bar{v}_{\theta} \bar{v}_{\theta}\right\rangle$ is dominated by VD, DS and PS, with VD and DS being dominant near the wall while PS being dominant in the region away from the wall. Note that PS holds always positive, i.e. draining the turbulent energy to the azimuthal fluctuation by the process of energy redistribution. The energy redistribution is the main mechanism to generate the azimuthal velocity fluctuation. It is interesting to notice that a non-zero PR arises in the $\left\langle\bar{v}_{\theta} \bar{v}_{\theta}\right\rangle$ budget due to the presence of the azimuthal mean flow in fig. 12(b). At $N=2$, PR becomes considerably important in the budget of $\left\langle\bar{v}_{\theta} \bar{v}_{\theta}\right\rangle$ near the wall region, owing to the augment of $\left\langle\bar{v}_{\theta} \bar{v}_{\theta}\right\rangle$ in the wall region, as shown in 
fig. 5(b). Compared to the non-rotating case, fig. 12(b) also reveals that the rotation effect can enhance both TD and VD, and PS contributes significantly to the generation of the azimuthal velocity fluctuation in the rotating pipe flow.
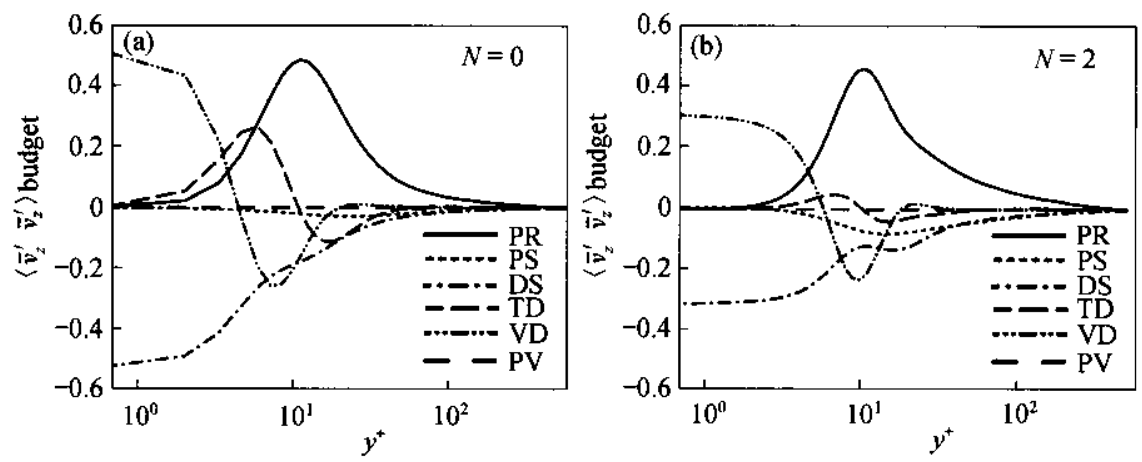

Fig. 11. Budgets terms in the transport equation of $\left\langle\bar{v}_{z} \bar{v}_{z}\right\rangle:$ (a) $N=0$; (b) $N=2$.
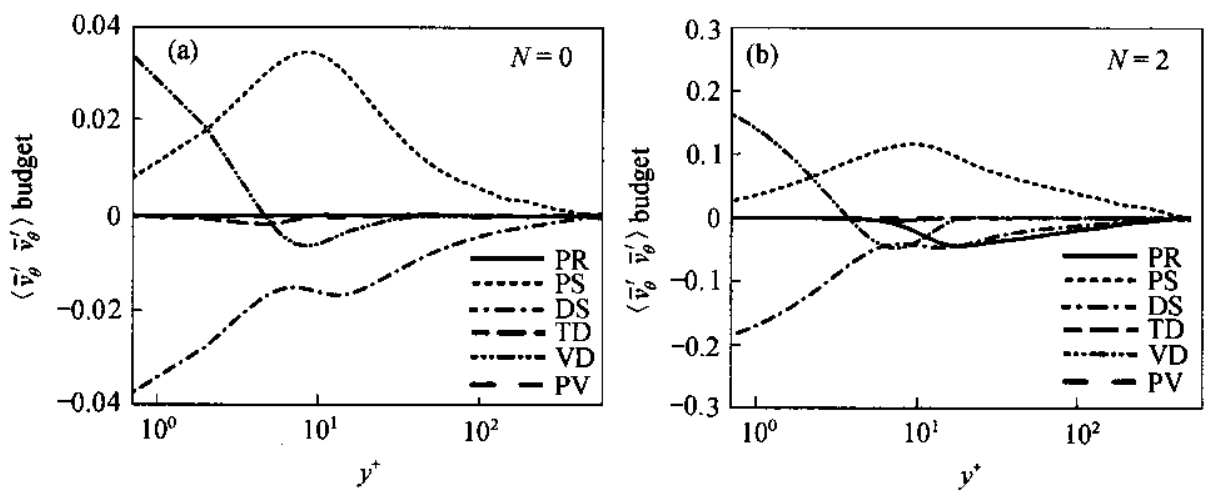

Fig. 12. Budgets terms in the transport equation of $\left\langle\bar{v}_{\theta} \bar{v}_{\theta}\right\rangle:$ (a) $N=0$; (b) $N=2$.

\subsection{Flow structures}

To exhibit the near-wall turbulence structures, the patterns of the axial velocity fluctuation $\bar{v}_{z}$ in the $(\theta, z)$ plane at $y^{+}=5.2$ are shown in fig. 13. As shown in fig. 13(a) for $N=0$, intensive high- and low-speed streaky structures appear. However, as exhibited in fig. 13(b) for $N=2$, the near-wall streaky structures are no longer aligned with the axial direction due to the rotation effect and depict obvious inclination with respect to the axial direction. The wall streaks are always aligned with the direction of the absolute mean flow. Thus, the impressing tilting of the streaky structures is due to the spiral motion of the fluid in the rotating pipe, since the rotational speed at the pipe wall induces the azimuthal mean flow which causes the absolute mean flow no longer parallel to the axial direction. According to Orlandi ${ }^{[4]}$, it is the tilting of the streaky structures that accounts for the decrease of the correlation between $\bar{v}_{r}$ and $\bar{v}_{z}$, and results in the drag reduction in the rotating pipe flow, as shown in fig. 5(a). 

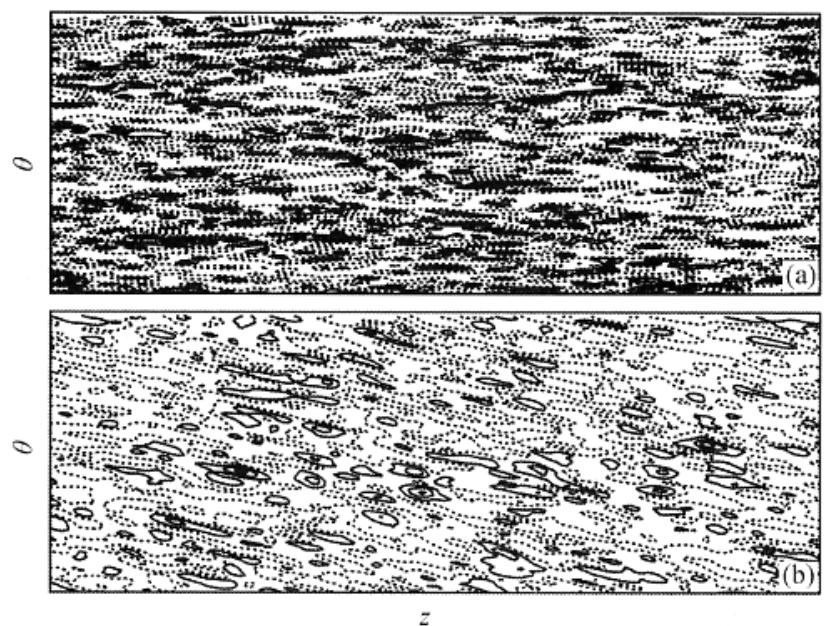

Fig. 13. Contours of the axial velocity fluctuation $\bar{v}_{z}$ in the $(\theta, z)$ plane at $y^{+}=5.2$ with the increment of contour 0.08: (a) $N=0$; (b) $N=2$.

Fig. 14 depicts the contour plots of the azimuthal velocity fluctuation $\bar{v}_{\theta}$ at $N=0$ and 2 in the same plane shown in fig. 13. The azimuthal fluctuations in the wall region at $N=0$ in fig. 14(a) are apparently weaker than those at $N=2$ in fig. 14(b). It is because that the generation of the azimuthal velocity fluctuation in the rotating case is mainly contributed by the enhanced process of energy redistribution and the shear effect due to the azimuthal mean flow. Meanwhile, fig. 14(b) exhibits the increased scale and strength of wall streaky structures linked to the azimuthal fluctuation. Similarly, the contours of $\bar{v}_{\theta}$ also indicate the remarkable redirection of the structures, which is an important feature of the rotating pipe flow.
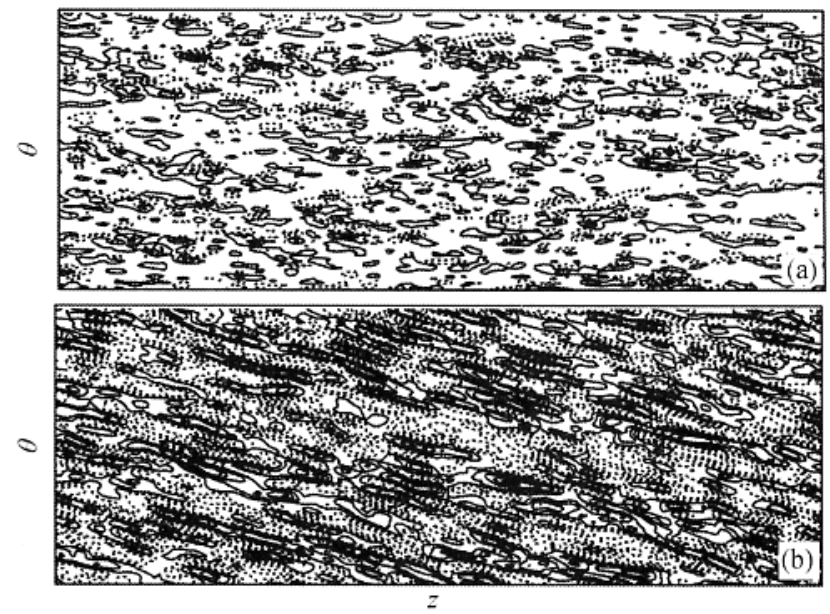

Fig. 14. Contours of the azimuthal velocity fluctuation $\overline{v_{\theta}}$ in the $(\theta, z)$ plane at $y^{+}=5.2$ with the increment of contour 0.03: (a) $N=0$; (b) $N=2$. 


\section{Concluding remarks}

By means of LES of turbulent flow through an axially rotating pipe, the rotation effect on the near-wall turbulence characteristics and flow structures is investigated. Some typical quantities, including the turbulence statistics, the helicity fluctuation, and the budgets of the resolved Reynolds stress, are analyzed in detail. The turbulent flow in the rotating pipe shows a tendency towards the laminar flow with the axial turbulence intensity suppressed. As the rotation number increases, the shear effect due to the azimuthal mean flow is enhanced, resulting in the higher value of the azimuthal turbulence intensity in the wall region. The joint PDFs of the velocity fluctuations reasonably predict that the drag reduction in the rotating pipe flow originates from weak correlation between the velocity fluctuations due to the rotation effect. The PDF of the helicity fluctuation implies that the more helical turbulence structures occur in the rotating pipe flow. By examining the budgets of the resolved Reynolds stress, it is found that the turbulent transport process is suppressed by the rotation effect, while the azimuthal turbulence fluctuation drains more kinetic energy by the process of energy redistribution. In the wall region of the pipe, the redirection of the high- and low-speed streaky structures is observed. Based on the information obtained by the present LES, it is of great help for the understanding of physical fundamentals in the rotating turbulent flows and for the development of reliable turbulence model.

\section{References}

1. White, A., Flow of a fluid in an axially rotating pipe, J. Mech. Eng. Sci., 1964, 6: 47-52.

2. Murakami, M., Kikuyama, K., Turbulent flow in axially rotating pipes, J. Fluids Eng., 1980, 102: 97-103.

3. Oberlack, M., Similarity in non-rotating and rotating turbulent pipe flows, J. Fluid Mech., 1999, 379: 1-22.

4. Orlandi, P., Fatica, M., Direct simulations of turbulent flow in a pipe rotating about its axis, J. Fluid Mech., 1997, 343: 43-72.

5. Imao, S., Itoh, M., Harada, T., Turbulent characteristics of the flow in an axially rotating pipe, Int. J. Heat Fluid Flow, 1996, 17: 444-451.

6. Reich, G., Beer, H., Fluid flow and heat transfer in axially rotating pipe 1. Effect of rotation on turbulent pipe flow, Int. J. Heat Mass Transfer, 1989, 32: 551-561.

7. Moin, P., Kim, J., Numerical investigation of turbulent channel flow, J. Fluid Mech., 1982, 118: $341-377$.

8. Rogers, M. M., Moin, P., Helicity fluctuation in incompressible turbulent flows, Phys. Fluids, 1987, 30: $2662-2671$.

9. Tsinober, A., Eggels, J. G. M., Nieuwstadt, F. T. M., On alignment and small scale structures in turbulent pipe flow, Fluid Dyn. Res., 1995, 16: 297-310.

10. Orlandi, P., Ebstein, D., Turbulent budgets in rotating pipes by DNS, Int. J. Heat Fluid Flow, 2000, 21: 499-505.

11. Mansour, N. N., Kim, J., Moin, P., Reynolds-stress and dissipation-rate budgets in a turbulent channel flow, J. Fluid Mech., 1988, 194: 15-44.

12. Wang, M. H., Fu, S., Zhang, G. H., Large-scale spiral structures in turbulent thermal convection between two vertical plates, Phys. Rev. E, 2002, 66: 066306-1-066306-8.

13. Tsinober, A., Turbulent drag reduction versus structure of turbulence, in Structure of Turbulence and Drag Reduction (ed. Gyr, A.), Berlin: Springer-Verlag, 1990, 313-340.

14. Orlandi, P., Helicity fluctuations and turbulent energy production in rotating and non-rotating pipes, Phys. Fluids, 1997, 9: 2045-2056.

15. Yang, Z. Y., Large eddy simulation of fully developed turbulent flow in a rotating pipe, Int. J. Numer. Method Fluids, 2000, 33: 681-694.

Copyright by Science in China Press 2005 
16. Smagorinsky, J., General circulation experiments with the primitive equations. I. The basic experiment, Mon. Weather Rev., 1963, 91: 99-112.

17. Germano, M., Piomelli, U., Moin, P. et al., A dynamic subgrid-scale eddy viscosity model, Phys. Fluids, 1991, A3: $1760-1765$.

18. Kosovic, B., Subgrid-scale modelling for the large-eddy simulation of high-Reynolds-number boundary layers, J. Fluid Mech., 1997, 336: 151-182.

19. Speziale, C. G., Subgrid scale stress models for the large-eddy simulation of rotating turbulent flows, Geophys. Astrophys. Fluid Dyn., 1985, 33: 199_222.

20. Kobayashi, H., Shimomura, Y., The performance of dynamic subgrid-scale models in the large eddy simulation of rotating homogeneous turbulence, Phys. Fluids, 2001, 13: 2350_2360.

21. Liu, N. S., Lu, X. Y., Zhuang, L. X., An improved dynamic subgrid-scale model for rotating turbulence and its application, Science in China, Ser. G, 2004, 47(4): 463-476.

22. Verzicco, R., Orlandi, P., A finite difference scheme for direct simulation in cylindrical coordinates, J. Comput. Phys., 1996, 123: 402-413.

23. Liu, N. S., Lu, X. Y., Large eddy simulation of turbulent concentric annular channel flows, Int. J. Numer. Methods Fluids, 2004, 45: 1317-1338.

24. Dong, Y. H., Lu, X. Y., Large eddy simulation of a thermally stratified turbulent channel flow with temperature oscillation on the wall, Int. J. Heat Mass Transfer, 2004, 47: 2109-2122.

25. Wang, L., Lu, X. Y., An investigation of turbulent oscillatory heat transfer in channel flows by large eddy simulation, Int. J. Heat Mass Transfer, 2004, 47: 2161-2172.

26. Wang, L., Dong, Y. H., Lu, X. Y., An investigation of turbulent open channel flow with heat transfer by large eddy simulation, Comput. Fluids, 2005, 34: 23-47.

27. Kim, J., Moin, P., Moser, R., Turbulence statistics in fully developed channel flow at low Reynolds number, J. Fluid Mech., 1987, 177: 133-166.

28. Kikuyama, K., Murakami, M., Nishibori, K., Development of three-dimensional turbulent boundary layer in an axially rotating pipe, J. Fluids Eng., 1983, 105: 154-160.

29. Speziale, C. G., Younis, B. A., Berger, S. A., Analysis and modelling of turbulent flow in an axially rotating pipe, J. Fluid Mech., 2000, 407: 1-26. 